\title{
Build on URLLC System with Modular Blocks
}

\author{
Huamin Fan \\ Nokia Corporation GCHN Region, Shanghai, China \\ Email: 1542590486@qq.com
}

How to cite this paper: Fan, H.M. (2021) Build on URLLC System with Modular Blocks. Journal of Computer and Communications, 9, 38-47. https://doi.org/10.4236/jcc.2021.912003

Received: June 3, 2021

Accepted: December 26, 2021

Published: December 29, 2021

Copyright (c) 2021 by author(s) and Scientific Research Publishing Inc. This work is licensed under the Creative Commons Attribution International License (CC BY 4.0).

http://creativecommons.org/licenses/by/4.0/

\begin{abstract}
The article focuses on the 3GPP key features URLLC and tells more about how to realize the uRLLC function to support more industrial scenarios. As the industrial application will require more specific and more detailed function set. The article creatively constructed the modular to design URLLC system from multi-dimensions. The system with modular blocks is a common methodology for planning the layout of future URLLC system commercial comprising which is helpful for the design and test scenarios. It is obvious that $5 \mathrm{G}$ network is developing rapidly from the basic high data throughput as the startup to high reliability and low latency. We are placing great hopes on it for future low-latency scenarios. The modular design methodology for improving the system realization and optimization will develop soon. The vision and method will help us open up more mindset to build promising $5 \mathrm{G}$ system for verticals.
\end{abstract}

\section{Keywords \\ eMBB, Critical URLLC TSN 5G NR}

\section{Content}

As we know, URLLC is one of the most attractive features of $5 \mathrm{G}$ in the new system. URLLC stands for Ultra Reliability and Low Latency Communication. We are placing great hopes on it for future low-latency scenarios. From the standard perspective, the three pillars of $5 \mathrm{G}$ features with reliability, latency and throughput have their own influences. It is obvious that $5 \mathrm{G}$ network is developing rapidly from the basic high data throughput as the startup to high reliability and low latency. Reliability \& latency have an inverse relationship with throughput. In some cases, we can choose high reliability \& low latency at the expense of high throughput. The classical scenarios, based on which 3GPP has specified the initial URLLC features, come from V2X, industry robotics and automation, electricity distribution, mobile tele-operation in drone and vehicles which all embo- 
dy the same characteristics of network latency lower than $50 \mathrm{~ms}$ and ultra-high reliability but small data. The use cases that benefit the most are those with deterministic packets. In summary, the unique advantage of URLLC can be listed as follows:

- ultra responsive connections.

- less than $1 \mathrm{~ms}$ air interface latency.

- $5 \mathrm{~ms}$ end to end latency between UE (i.e. mobile) and 5G eNB (i.e. base station).

- ultra-reliable and available $99.9999 \%$ of the time.

- low to medium data rates (about $50 \mathrm{kbps}$ to $10 \mathrm{Mbps}$ ).

- high speed mobility.

On the other hand, the market demand is also driving the URLLC ready and matured for deployment. Once the network is enabled from eMBB-capable to URLLC-driven, enterprises and CSPs are more likely to introduce URLLC based use cases (just as Figure 1 shows) once the balance can be achieved between eMBB capacity and low latency.

Manufacturing, electric-power, transportation and entertainment all propose use cases performances mapping to URLLC, like the factory automation, critical process automation, electricity distribution, transportation automation which all distinguished different URLLC levels according to the degree of serve demand level from Non-critical URLLC, Non-critical URLLC, Moderate critical LLC, Moderate critical URLLTSC/URLLC, Critical URLLTSC/URLLC, Highly critical URLLTSC.

It is important to remember that each network element and the architecture structure contribute to Latency budget: improvements on Air interface performances are tangible only if the full e2e network is optimised. Therefore, in addition to radio features, it is essential to assess quality of the transport network and where the application is placed.

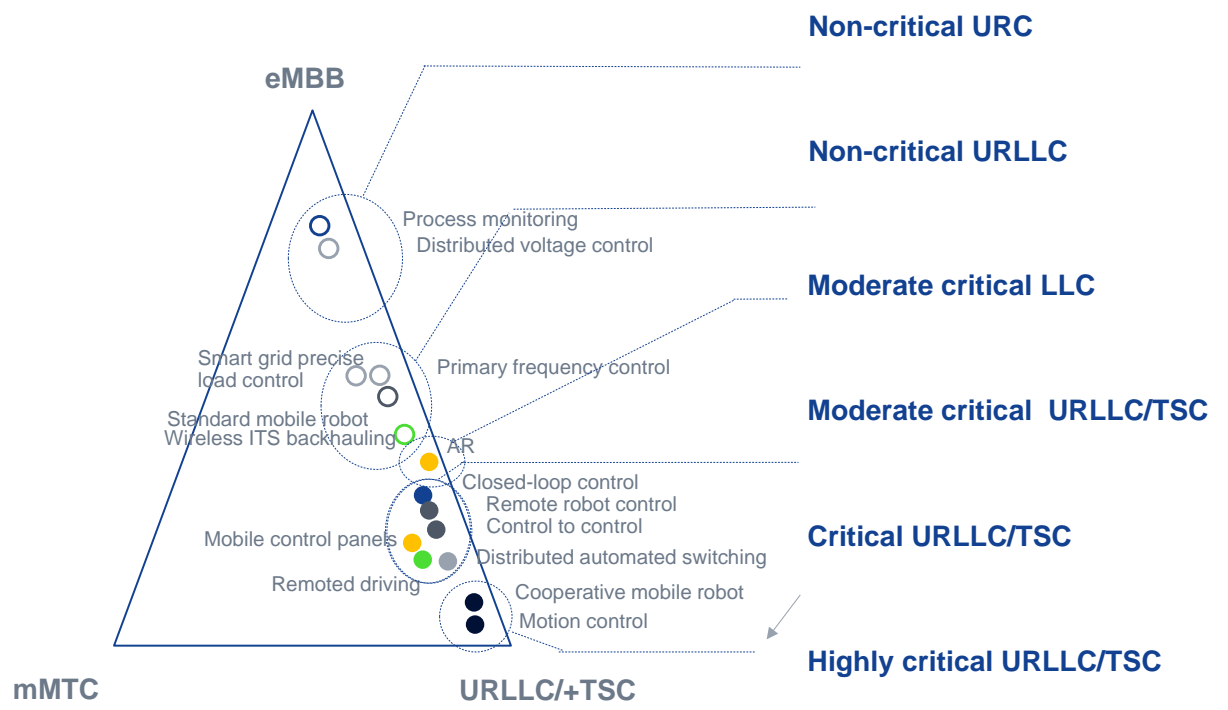

Figure 1. 3 key features of $5 \mathrm{G}$. 
Moreover, system parameters, like TDD/FDD domain, frequency, modulation, etc., scenario parameters like indoor/outdoor, propagation factor and use cases traffic model may impact the overall performances.

System dimensioning and traffic conditions influence the URLLC performances results; the degree of freedom to select the features to deliver the requirements is limited by system configurations and deployment scenario.

Let's take a look at the 3GPP standards relevant to URLLC. 3GPP Rel15 RAN specs support URLLC service for small data payloads (e.g. 32 B - 200 Byte) with radio latency of $1 \mathrm{~ms}+$ and with an outage probability of less than 10-5 [1].

\section{Key Features Include}

- Mini slot and new numerology

Mini-slot consists of two or more symbols, the first of which contains control information. 2/4/7 symbol scheduling. Mini slot supported in 5G NR not like 14 symbols in LTE system will reduce data transmission and feedback latency time with 2/4/7 symbol transmissions independent of slot length.

- Uplink configured grant

The user (UE) can be configured to transmit in grant-free/configured-grant (GF/CG) resources for uplink (UL) transmission that does not require the UE to transmit scheduling request (SR) and receive UL grant to reduce latency.

- DL pre-emptive scheduling

Effective scheduling to reduce DL latency, latency critical data is immediately scheduled by puncturing the ongoing transmission to UE rather than waiting for its completion, i.e. avoiding additional latency.

- PDCP layer data duplication

PDCP duplication is a low complexity technique since it exploits independent transmissions of the copies (e.g. independent scheduling and link adaptation) which in Rel.15 increases radio resource usage resulting in higher interference and queuing delay.

- Flexible frame structure

5G NR supports LTE system $15 \mathrm{KHz}$ carrier spacing, but also supports more space solutions including $30 \mathrm{KHz}, 60 \mathrm{KHz}, 120 \mathrm{KHz}, 240 \mathrm{KHz}$, the higher the carrier spacing brings lower latency performance; At the same time, 5GNR supports adjusting frames structure. NR can switch between time slots 1, 2, and 4 flexibly and can configure the upstream and downstream ratio flexibly, which greatly reduces the delay.

- Enhanced scheduling

Reduce delay by eliminating handshake required by dynamic scheduling between UE/gNB for uplink traffic. No waiting time is needed anymore (with resource aligned with traffic arrival).

- Flexible PDCCH configuration/PDCCH enhancements

There are two ways to support PDCCP enhancement configuration. One is DCI format(s) with configurable sizes the other is Increased PDCCH monitoring 
capability.

Reasonable configuration of PDCCH monitoring period and offset value as well as PDCCH monitoring pattern in a time slot can realize relatively intensive PDCCH monitoring opportunities. There are multiple PDCCH monitoring moments in a time slot, which can respond to URLLC's sudden burst business scenarios and meet the requirements of low delay.

- URLLC higher priority transmission

The characteristics of URLLC data in low-delay scenarios are mainly strong suddenness but small data volume, so NR supports URLLC to occupy channel resources in a preemptive way. When the base station allocates physical resources to the eMBB service, it allocates the resources of the eMBB service to the URLLC service at the same time. When the URLLC preempts the physical resources, NR notifies the UE of the preemptive result to ensure the low latency requirements of the URLLC.

- MEC technology

$5 \mathrm{G}$ network can sink the UPF functions to the user side, and the edge computing server and UPF are co-deployed. When UPF recognizes that the destination address of the business flow is local, it will be shunt to the local edge computing server for business processing, which reduces the redundant transmission path of the business and reduces the delay.

In the phase of R16, more 3GPP URLLC Low Delay Enhancement Solution are forwarded. 3GPP Rel16 and beyond introduces advanced techniques and new technical enablers to support more stringent requirements:

1) Authorization free configuration-UL configuration grant: The base station is pre-configured with periodic resources, so UE does not need to apply to the base station. UE applies to the base station in advance for the resources used by PUSCH and configures the corresponding parameters. When uplink resources are available, these resources are directly used for transmission, saving the time to send scheduling requests to the base station, apply for resources and receive feedback from the base station, and ensuring the low latency requirements of URLLC. On the other hand, this mechanism might impact the overall network capacity as resources are preassigned to uplink even when there is nothing to be transmitted.

2) HARQ enhancement: In R15, UE can only transmit HARQ-ACK on PUCCH once in a time slot. When the UE needs to issue HARQ-ACK again on a PUCCH in the same slot in order to reduce the delay, this is not allowed. In the R16 phase, HARQ-ACKS are allowed to be fed back over multiple PUCCH channels within a time slot. To support this design, the R16 terminal requires that the UE support at least two HARQ encoding modes and that the physical layer is recognized.

3) TSN and URLLC combination: Realize time sensitive transmission to ensure clock synchronization. High precision reference clock can be broadcast in $\mathrm{PBCH}$ or sent in RRC layer to ensure the precise time synchronization between 
master clock and terminal clock and realize time-sensitive transmission.

Actually, with the development of 3GPP standards, more features will be supplemented which all enrich the industrial scenarios demand, we are trying to use the URLLC system modular blocks (as shown in Figure 2) to demonstrate a specific use case which will help to boost future system setup. Building and selecting blocks depends on application scenario and will require trade-offs between reliability, latency and throughput requirements. The blocks will be composed of as follows:

To achieve the best performance under URLLC system with the balanced features of high reliability and capacity, it is still a lot of work to do. But specification is developing and evolving. Extreme Latency can come at the cost of the reliability and capacity (you can refer to Figure 3: the balance and impact among latency, reliability and capacity) and Optimizing for Ultra Reliability can also come at the cost of the latency and capacity of the system. In cost, we should over-dimension the network and make the use case on a certain scale to achieve the economic result. No operator will be available to overspend for few and limited use cases.

Scalable numerology Mini slots URLLC preemption Short PUCCH UL configured grant Scalable numerology QoS differentiation SPS for DL Reduced processing time Mini slot PUCCH Inter UE priority PDCP duplication Slot aggregation Robust MCS

Figure 2. Key feature blocks of URLLC.

Latency vs. Reliability vs. Capacity impact

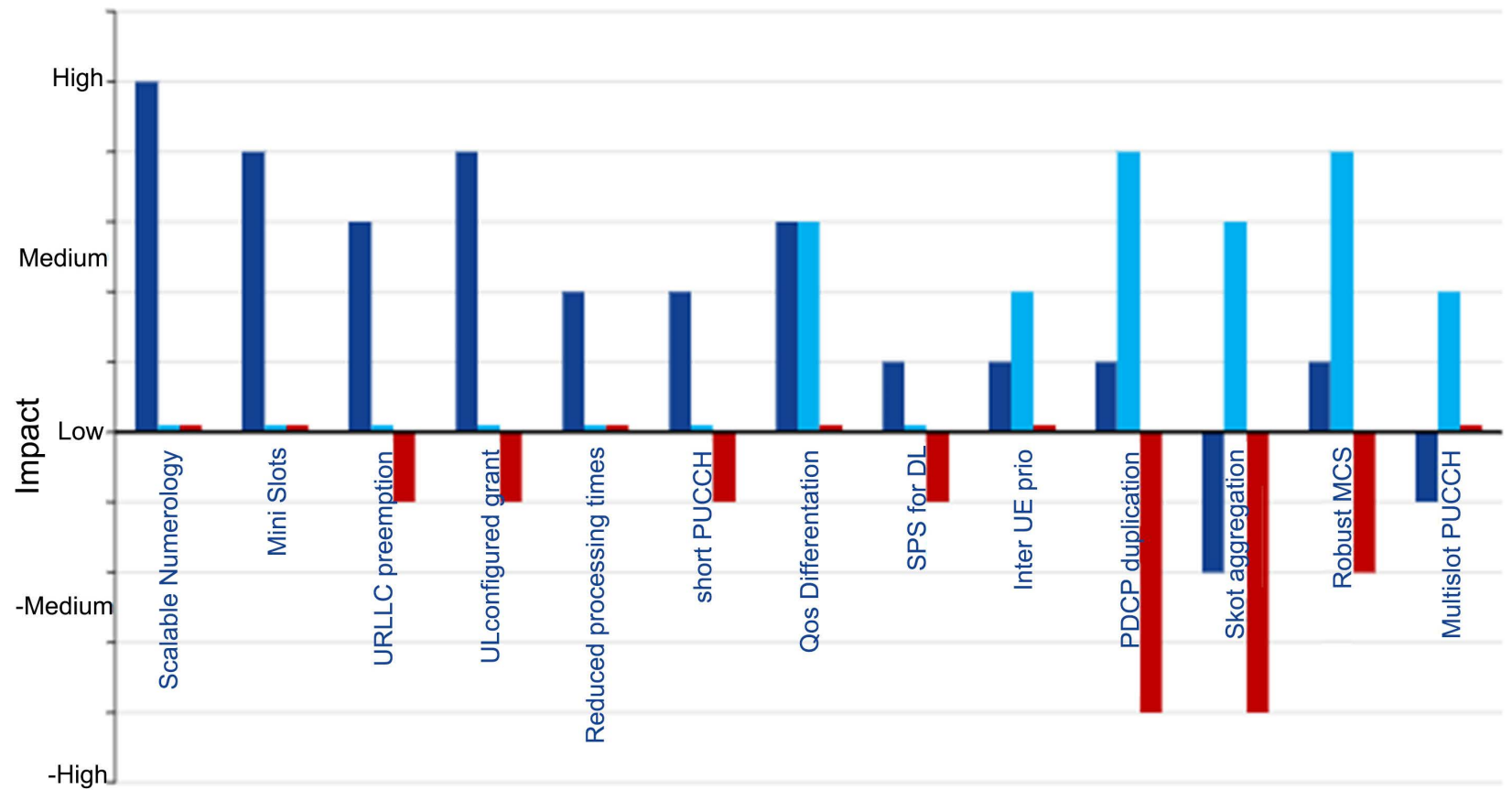

- Latency $=$ Reliability " Capacity

Figure 3. The balance and impact among latency, reliability and capacity. 
How to build a prosperous and feasible URLLC network becomes more and more challenging. Modular Setup method can help us understand a use case's requirements and deployment demand more easily which will lead to future launch possibly.

In order to explain how to select the feature set, let us set up 4 dimensions which are use case requirement, system configuration, latency toolbox, reliability toolbox to create a URLLC modular coreset which incorporate the optimal features combinations system parameters can define (refer to Figure 4: requirements from 4 pilliars).

Then we can select the classical and specific modular to set up a use case from the 4 pillar perspectives [2] (Figure 5).

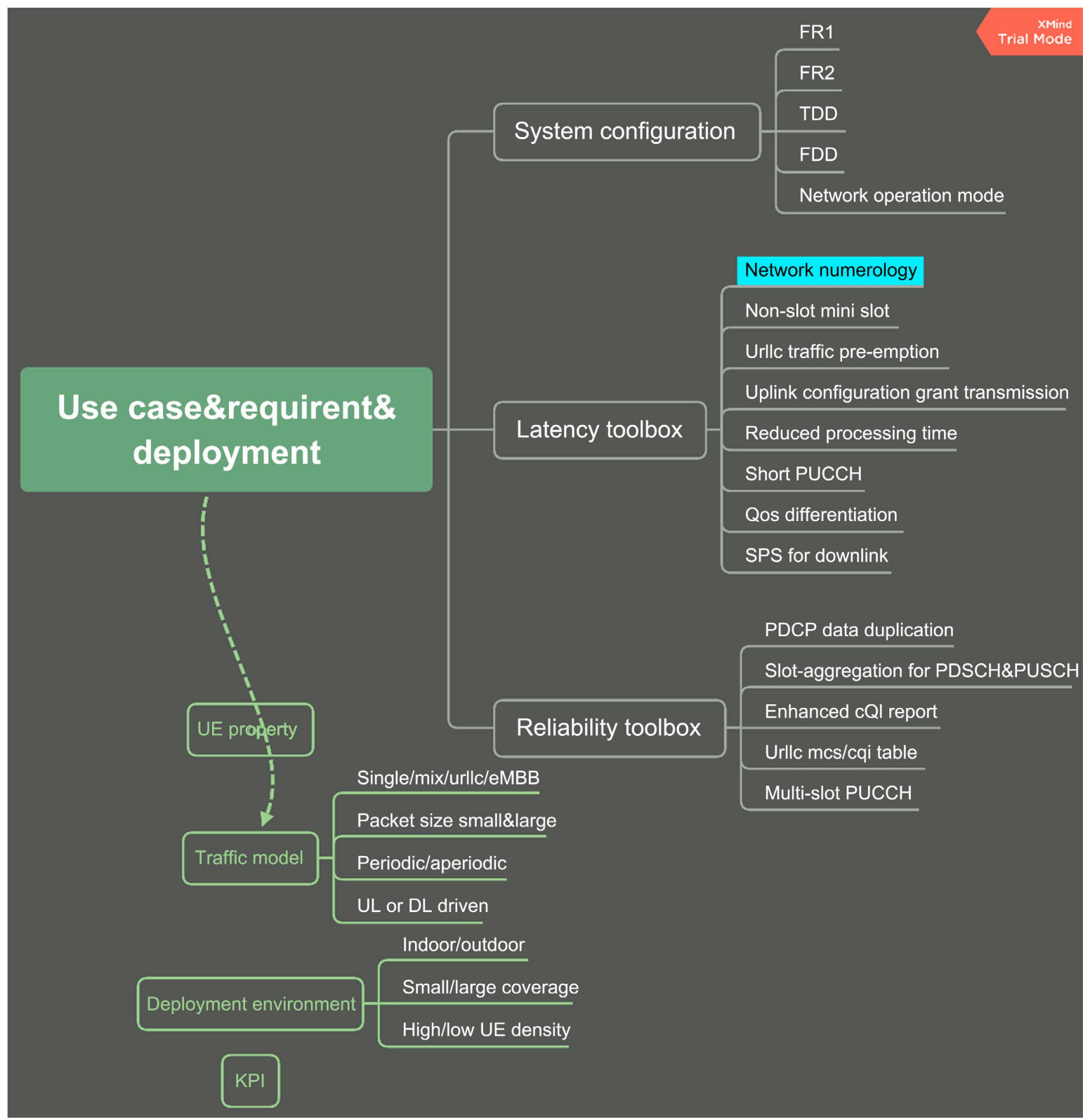

Figure 4. Use case requirement from 4 pillar perspective. 


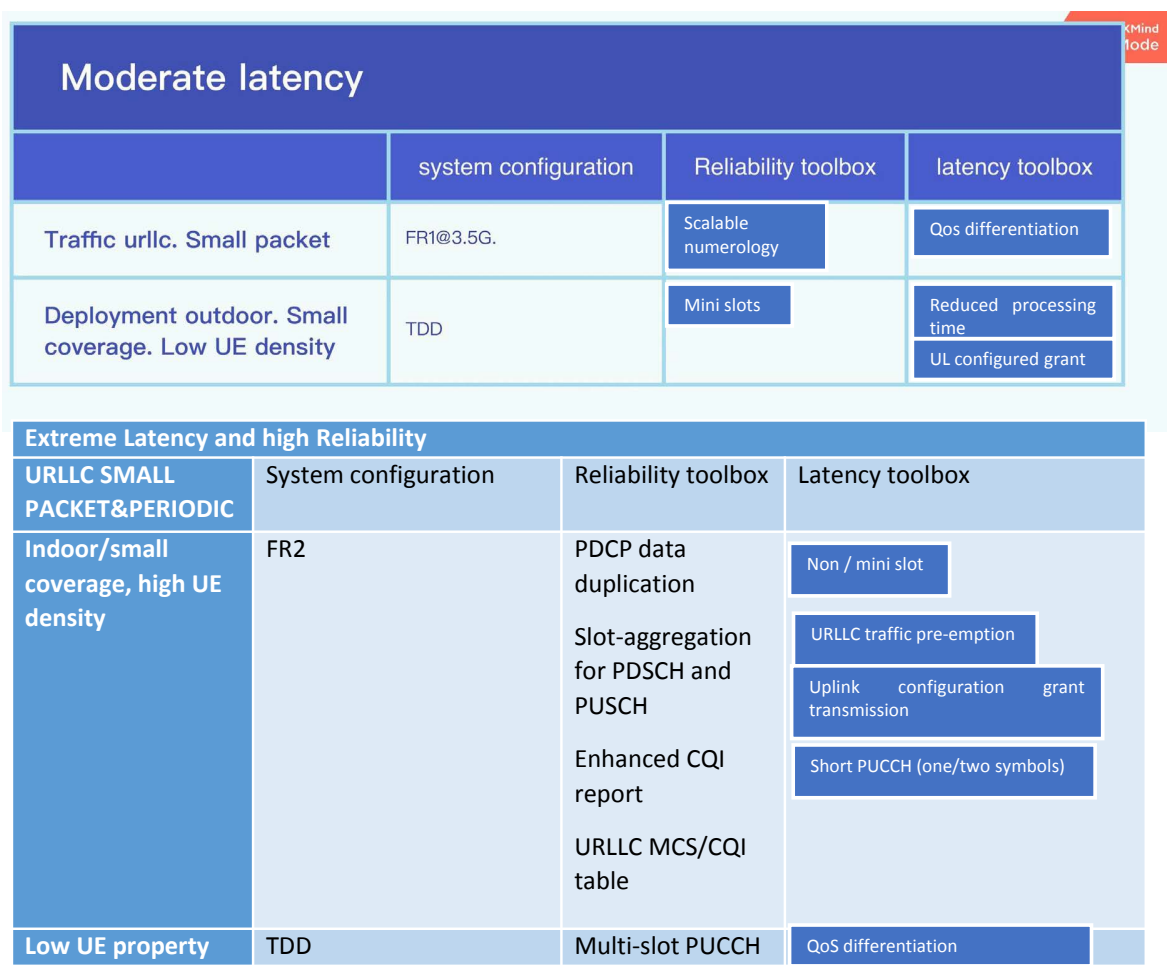

Figure 5. Different latency selection.

Based on the summary of URLLC system with modular blocks theories, we can have the ideal application example to test it.

There is a cooperative example for professional audio transmission with Sennheiser. The case comes from Nokia Bell Labs which we are studying on [3]. The Audio/Media industry is exploring to replace proprietary RF technology in future professional wireless audio systems with $5 \mathrm{G}$ technology and extend the possible range of applications beyond the classical audio/video. The pain point for the artists in the stage is mainly wireless connection and audio transmitter quick feedback for users. Here the key point will be how to capture, produce, playback, and distribute audio content in an efficient way. The typical live audio production setups rely on the wireless and wireline technologies. When we try to overturn traditional technology with 5G, the URLLC system will come in handy with its unique characteristics. In this scenario, there are three requirements: wireless transmission latency $<1 \mathrm{~ms}$, reliability $99.9999 \%$, synchronicity $<1$ us. So we can tell concrete system configuration to achieve the Audio requirements like below (Figure 6):

\section{Main config parameters}

- $3.5 \mathrm{GHz}$

- $100 \mathrm{MHz} \mathrm{BW}$

- TDD Duplex

- $24 \mathrm{bit} / \mathrm{sample}$ at $48 \mathrm{kHz}$ sample/rate

- ADC audio signals

- Proprietary sample compression 


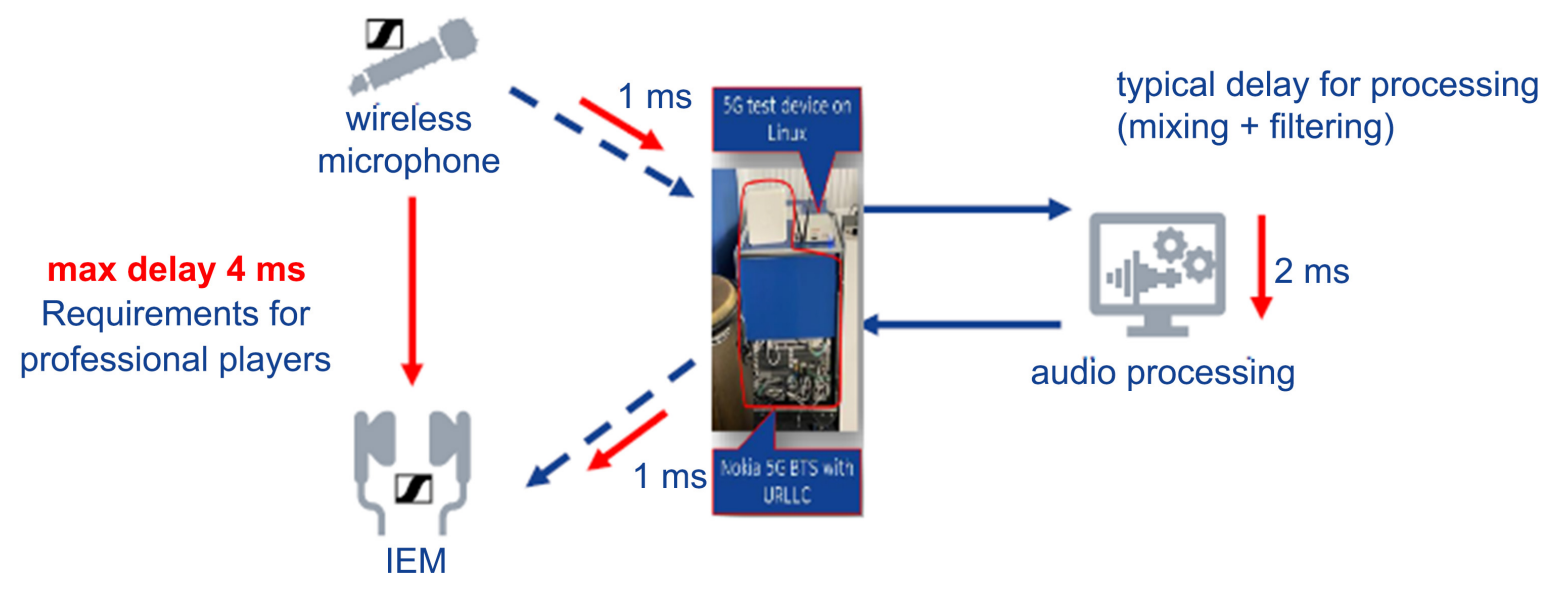

Figure 6. An example of ideal application.

\section{URLLC features applied}

- $30 \mathrm{kHz}$ SCS numerology

- 2-symbols mini-slot support for UL and DL

- Mini-slot based scheduling every $0.5 \mathrm{~ms}$ for downlink and uplink

- Uplink grant-free transmission

- DL scheduling based on Downlink Control Information (DCI)

- Packet core and application processing were co-located with the system module for baseband processing in order to minimize latency impacts

Detailed latency split in the process (refer to Figure 7: detailed latency split process). In this case, from audio packet sender to audio packet receiver, the main packet-transferring time in $5 \mathrm{G}$ system will consist of the following processing steps. Through the URLLC optimal and optional features combinations, we can do it to make the best audio performance and experiences to make the concert and other live stadium scenarios more perfect in the future.

Of course, Modeling was very realistic, starting from measurements. Scenario parameters and use cases were defined together with industry partner which needs us more flexible and probing into any possible use case. Let's do it down-to-earth with the URLLC standards more matured. The industrial IoT time will come soon, URLLC time will show up consequently as well.

\section{Challenge for URLLC Technology and Market Facing}

Although we can see promising future for URLLC, currently, there are still huge challenges for 5G URLLC application in standards, frequency, network deployment, terminals and business modes etc.

- Standard [4] [5]

Just as we learned 3GPP R15 mainly defines eMBB specifications, what we are expecting URLLC in 5G revolutionary technology will be ready in R16 and so on. Commercial deployment and industry supply chain maturity also need several years after the standard is determined to achieve commercial scale.

- Frequency and network [6] 


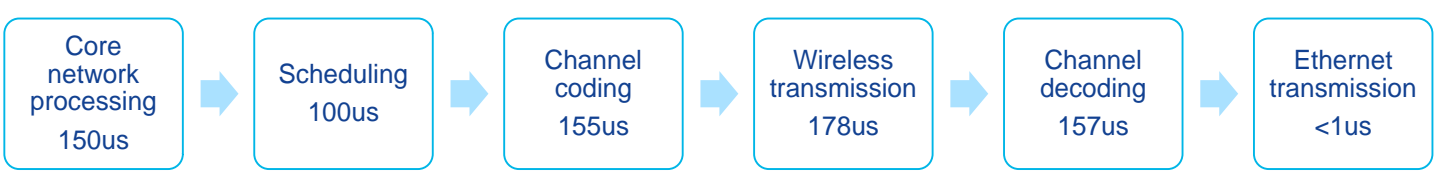

Figure 7. Detailed latency split process.

At present, 5G in the world is mainly used in eMBB business. When introducing ultra-low delay, we need to balance eMBB and URLLC services. If we only consider the deployment of critical/Ultra Critical URLLC services, it may be difficult to apply them on the same physical network at the same time. Even if the virtual private network adopts the network slicing method, the network operation and maintenance may become complicated due to the huge differences in application scenarios. Therefore, operators may need to consider using dedicated frequency bands and physical networks to deploy URLLC, such as FDD spectrum refarming.

URLLC can be applicable for all kinds of scenarios, but each is different, such as coverage size, mobility, latency, throughput, reliability, different KPI will result in different URLLC deployment, which makes the spectrum strategy and network deployment of URLLC complex and difficult to balance.

Besides that, the terminals for URLLC will also be diversified in vertical industry. Some terminals are in pursuit of performance, some pursue power consumption and cost, and the application requirements of various industries are not the same. Therefore, the product form of terminals will present the trend of diversification and customization, which also puts forward new requirements for the maturity cycle of the industrial chain.

- Business mode [7] [8]

It is confirmed that URLLC will be more applied to future vertical industries, the application scenarios, and requirements of all walks of life are not the same. As service providers, they should customize and set flexible strategies and interfaces according to the characteristics of the industry. Although 5G introduces network slicing architecture technology and customizes more standardization to make it more adaptable for vertical industry application, business model and operation and maintenance are still full of new and unpredictable challenges. No matter telecom operators, Internet companies, system equipment providers and terminal manufacturers, they all need to cooperate together and unite all parties in the industry circle to carry out the all-round ecological construction of 5G URLLC, demonstrate the effect of 5G URLLC business landing, and promote the continuous growth of 5G URLLC ecosystem.

\section{Summary}

Low latency is seen as a powerful newly adding ingredient in 5G to ensure applications are usable and interactive whether communication is human-to-human, human-to-machine or machine-to-machine. Compared with $4 \mathrm{G}$, we can see the upcoming feature has outstanding in the standards development growing out of 
nothing. Design, standardization and engineering challenges are being overcome to deliver networks that are both reliable and provide low latency.

As we can identify the URLLC scenarios demanding features in detail, modular setup method will help us to grasp the key KPIs and modular elements to create an ideal target network. Here, we only selected one entertainment case which has improved the application effect through building up URLLC modular network. Actually, there will be more and more cases suitable for it. The upcoming use cases of URLLC in tele-surgery, smart transportation and industry automation, and etc. all present the latency and reliability requirements for these application scenarios. The key latency bottlenecks in current cellular network still need us to overcome and improve. We need to study further emerging applications, design challenges, and potential approaches in the design of URLLC based on 3GPP r16/17 standards and modular setup modes. It is a long way, but it is convinced that URLLC is a correct and promising way for us to follow.

\section{Conflicts of Interest}

The authors declare no conflicts of interest regarding the publication of this paper.

\section{References}

[1] 1.5G Americas (2018) 5G_Americas_URLLC_white_paper_final_11.8.

[2] Zhu, H. and Lin, Y. (2017) Research on 3GPP URLLC Standard, Key Technique and Network Architecture for 5G.

[3] https://wenku.baidu.com/view/733be0c486c24028915f804d2b160b4e777f81ed.html? $\underline{\mathrm{fr}}=$ search-1-wk user vipX-income5\&fixfr=O555DD2gGtcWN4u8WfD6KQ\%3D\% $\underline{3 \mathrm{D}}$

[4] Bell Lab. (2021) Low Latency 5G for Professional Audio Transmission. https://www.bell-labs.com/institute/white-papers/low-latency-5g-professional-audi o-transmission/

[5] China Unicom Network Technology Research Institute (2019) China Unicom 5G URLLC Technical White Paper.

[6] What's a Time Crystal? And How Do Google Researchers Use Quantum Computers to Make Them? (2021). https://spectrum.ieee.org

[7] 3GPP (2020) 3GPP TS 38.211: NR; Physical Channels and Modulation.

[8] China Mobile Research Institute (2020) China Mobile CMRI Wireless Network Capability White Paper Targeting at URLLC Scenarios. 